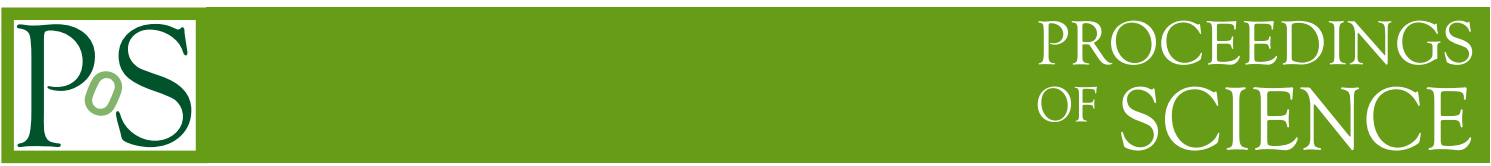

\title{
Low-mass dilepton measurements with ALICE at the LHC
}

\author{
Alberto Calivà* on behalf of the ALICE Collaboration \\ GSI Helmholtzzentrum für Schwerionenforschung $\mathrm{GmbH}$ \\ E-mail: alberto.calivadcern.ch
}

\begin{abstract}
Dileptons are unique probes to study the space-time evolution of ultrarelativistic heavy-ion collisions since they are produced continuously and carry unaffected information on the production source. The dilepton invariant-mass spectrum is sensitive to in-medium effects of low-mass vector mesons, connected to the partial chiral symmetry restoration, and to thermal radiation emitted in the partonic and hadronic phases. The study of dilepton production in pp collisions provides a fundamental baseline for the measurement in heavy-ion collisions and allows for the tuning of light particle production models at LHC energies.

The final results of the dielectron measurement in $\mathrm{Pb}-\mathrm{Pb}$ collisions at $\sqrt{s_{\mathrm{NN}}}=2.76 \mathrm{TeV}$ and the preliminary results in $\mathrm{Pb}-\mathrm{Pb}$ collisions at $\sqrt{s_{\mathrm{NN}}}=5.02 \mathrm{TeV}$ are presented. The preliminary results of the $\phi$-meson production measurement in pp collisions at $\sqrt{s}=5.02 \mathrm{TeV}$ using the dimuon channel, and the center-of-mass energy dependence of its production cross section in pp collisions are also shown.
\end{abstract}

International Conference on Hard and Electromagnetic Probes of High-Energy Nuclear Collisions 30 September - 5 October 2018

Aix-Les-Bains, Savoie, France

${ }^{*}$ Speaker. 


\section{Introduction}

Dileptons, i.e., lepton-antilepton pairs, are produced continuously during all stages of the spacetime evolution of ultrarelativistic heavy-ion collisions. These particles interact with the hot and dense system created in these collisions only through the electroweak force, thus carrying essentially unperturbed information on their production source. The dilepton invariant-mass spectrum is used to study physical processes occurring at different stages of the collision. The low-mass region of the dilepton spectrum $\left(m_{1^{+} 1^{-}}<m_{\phi}\right)$ is sensitive to thermal radiation, emitted during the partonic and hadronic phases, and to in-medium modifications of low-mass vector meson spectral functions, which are connected to the partial chiral symmetry restoration at high temperatures $[1,2]$. In the intermediate-mass range $\left(m_{\phi}<m_{1^{+} 1^{-}}<m_{J / \psi}\right)$, the thermal radiation from the Quark-Gluon Plasma (QGP) and the contribution from semi-leptonic heavy-flavor decays can be studied. The latter is expected to be partially suppressed in nucleus-nucleus collisions with respect to proton-proton (pp) collisions due to the energy loss of heavy quarks in the medium and cold nuclear matter effects. The intermediate mass range of the dilepton spectrum measured in pp collisions can be used to extract the production cross section of heavy quarks, as done in $[3,4]$, and to study the pair angular correlations of the heavy quarks produced in the initial hard parton-parton scattering, providing important information on the production mechanism of heavy quarks. Furthermore, the study of dilepton production in $\mathrm{pp}$ collisions provides a fundamental baseline for the measurement in heavyion collisions and allows for the tuning of light particle production models at LHC energies.

\section{Experimental apparatus and analysis techniques}

The ALICE apparatus and its performance are described in $[5,6]$. The dielectron measurement in $\mathrm{Pb}-\mathrm{Pb}$ collisions at a center-of-mass energy per nucleon pair $\sqrt{s_{\mathrm{NN}}}=2.76 \mathrm{TeV}$ is done in the centrality range $0-10 \%$, while that in $\mathrm{Pb}-\mathrm{Pb}$ collisions at $\sqrt{s_{\mathrm{NN}}}=5.02 \mathrm{TeV}$ uses $0-20 \%$. The centrality is selected based on the measured multiplicity in the V0 detectors [7]. Electrons are identified in the pseudorapidity range $\left|\eta_{\mathrm{e}}\right|<0.8$ using the measurement of the specific energy loss in the Time Projection Chamber (TPC) [8] and in the Inner Tracking System (ITS) [9], complemented by the time-of-flight measurement provided by the TOF detector [10]. The minimum electron transverse momentum $\left(p_{\mathrm{T}}\right)$ is $0.4 \mathrm{GeV} / c$ for the dielectron analysis in $\mathrm{Pb}-\mathrm{Pb}$ collisions at $\sqrt{s_{\mathrm{NN}}}=2.76 \mathrm{TeV}$ and $0.2 \mathrm{GeV} / c$ for that in $\mathrm{Pb}-\mathrm{Pb}$ collisions at $\sqrt{s_{\mathrm{NN}}}=5.02 \mathrm{TeV}$. The combinatorial background is estimated using the invariant-mass distribution of like-sign pairs, corrected for acceptance effects using the event mixing technique. Muons are identified in the pseudorapidity region $-4<\eta_{\mu}<-2.5$ in a dedicated forward spectrometer [5]. The combinatorial background in the dimuon analysis is estimated using the invariant-mass distribution of unlike-sign muon pairs from mixed events, normalized to the like-sign spectrum. The contribution from light-meson decays is estimated from a fit with mass shapes given by simulations.

\section{Dielectron results}

The dielectron invariant-mass spectrum measured in central $(0-10 \%) \mathrm{Pb}-\mathrm{Pb}$ collisions at $\sqrt{s_{\mathrm{NN}}}$ $=2.76 \mathrm{TeV}$ [11] is shown in Fig. 1 in comparison with the expected contributions from hadronic 
sources (left) and with the predictions from two theoretical model calculations that include, in addition, thermal dielectrons and in-medium modified $\rho^{0}$ (right) $[1,2,12]$.
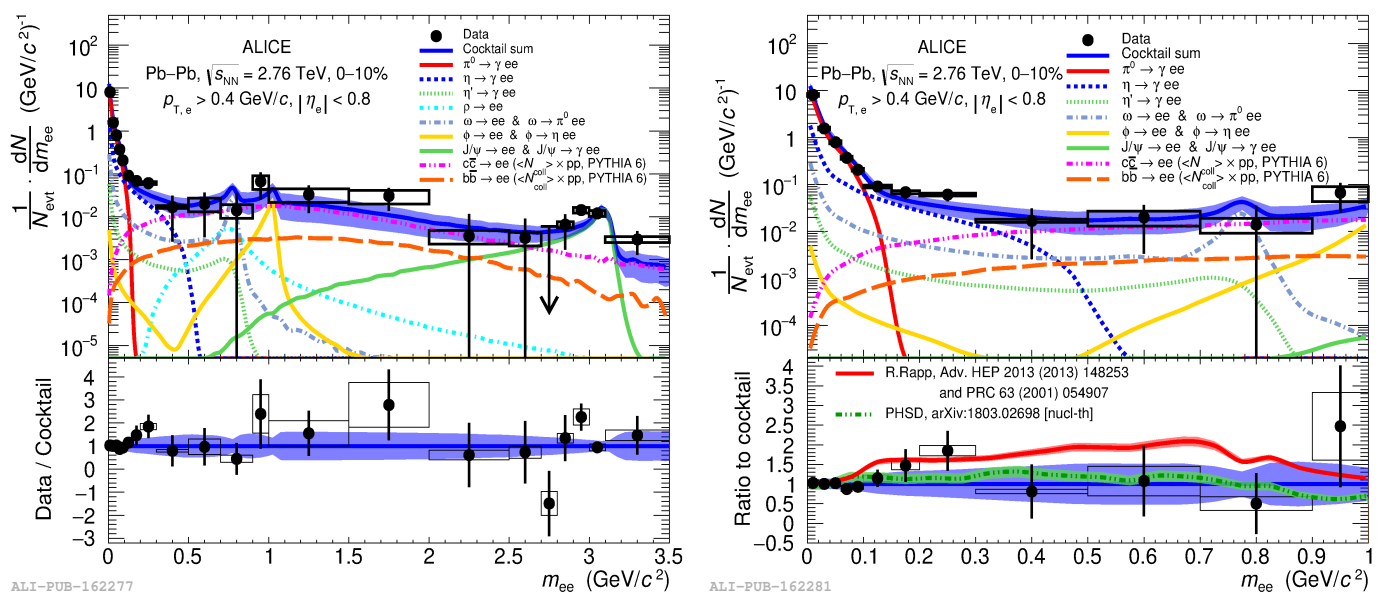

Figure 1: (colour online). Left: Dielectron invariant-mass spectrum measured in central $(0-10 \%) \mathrm{Pb}-\mathrm{Pb}$ collisions at $\sqrt{s_{\mathrm{NN}}}=2.76 \mathrm{TeV}$ compared to the hadronic cocktail. The statistical and systematic uncertainties of the data are represented by vertical bars and boxes. The arrow for one mass bin represents the upper limit at $90 \%$ confidence level. The blue band represents the systematic uncertainties on the hadronic cocktail. Right: Dielectron invariant-mass spectrum for $m_{\mathrm{ee}}<1 \mathrm{GeV} / c^{2}$ in comparison with the predictions from R. Rapp [1, 2] and PHSD [12] theoretical models.

The ratio of data and the hadronic cocktail, excluding the contribution from the vacuum $\rho^{0}$, is calculated in the invariant-mass range $0.15<m_{\mathrm{ee}}<0.7 \mathrm{GeV} / c^{2}$, where an excess of the dielectron yield was observed with respect to the hadronic cocktail in previous dilepton experiments [13-18]. Its value is $R=1.40 \pm 0.28$ (stat.) \pm 0.08 (syst.) \pm 0.27 (cocktail). The limited sensitivity of the dielectron measurement in the low-mass region prevents any analysis of a possible excess spectrum. The predictions from both theoretical models are consistent with the data within the experimental uncertainties. In the intermediate-mass region, the data are consistent with the hadronic cocktail which is based on binary scaling of the charm and bottom contributions. This indicates the limited sensitivity of this measurement to shadowing and energy loss effects of heavy quarks. In contrast, a clear suppression of dielectrons from heavy-flavor hadron decays is observed in the intermediatemass range of the dielectron spectrum measured in $\mathrm{Pb}-\mathrm{Pb}$ collisions at $\sqrt{s_{\mathrm{NN}}}=5.02 \mathrm{TeV}$. This is shown in Fig. 2 in comparison with the hadronic cocktail constructed assuming binary scaling of the charm and bottom contributions (left), and to a modified version of the cocktail which includes an estimate of shadowing effects for dielectrons from charm obtained based on the EPPS16 parametrization [19].

\section{Dimuon results}

The $\phi$-meson production cross section is measured in pp collisions at $\sqrt{s}=5.02 \mathrm{TeV}$ with improved statistical precision with respect to the existing results by including an additional data set, recorded in 2017. The systematic uncertainties are also reduced compared to the existing measurement thanks to improved signal extraction techniques. This measurement is shown in Fig. 3 left in 

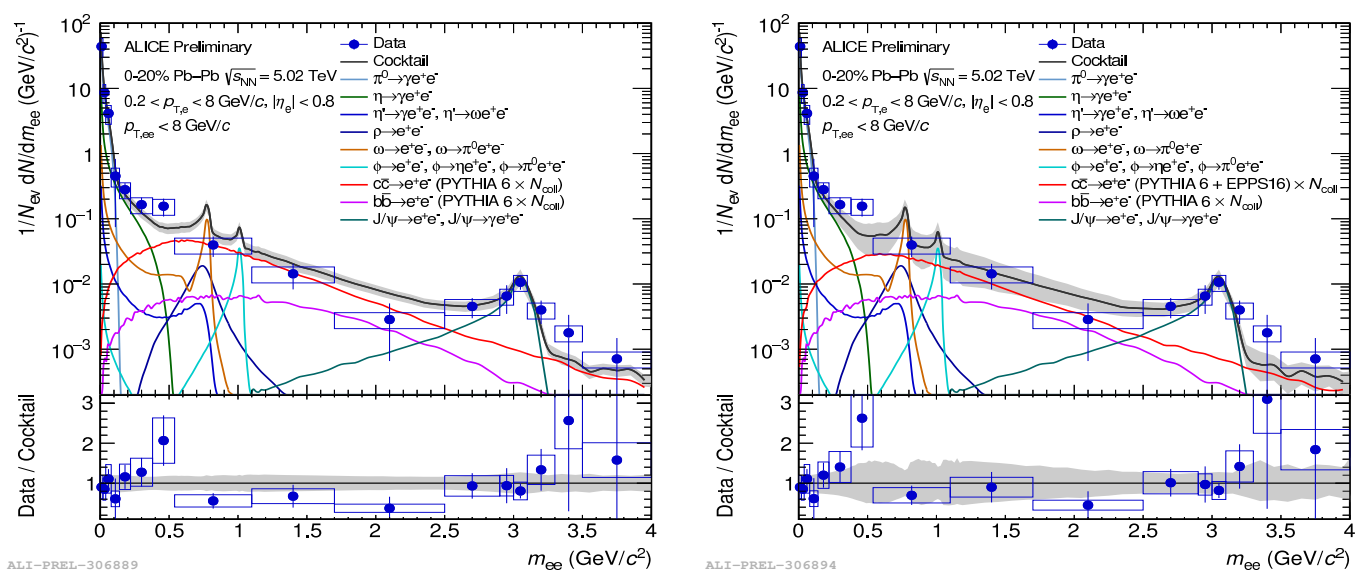

Figure 2: (colour online). Left: Dielectron invariant-mass spectrum measured in central $(0-20 \%) \mathrm{Pb}-$ $\mathrm{Pb}$ collisions at $\sqrt{s_{\mathrm{NN}}}=5.02 \mathrm{TeV}$ compared to the hadronic cocktail. The contributions from heavy-flavor decays are obtained assuming binary scaling. Right: Dielectron invariant-mass spectrum in comparison with the hadronic cocktail with modified charm obtained based on the EPPS16 parametrization [19].

comparison with the expectations from Phojet [21] and Pythia 8.1 with Monash-2013 tune [20]. While the data are fairly described by Phojet in the full $p_{\mathrm{T}}$ range, they are underestimated by Pythia by $\sim 50 \%$. The center-of-mass energy dependence of the $\phi$-meson production in pp collisions is shown in Fig. 3 right. The hardening of the $p_{\mathrm{T}}$ spectrum of the $\phi$ meson with increasing center-ofmass energy is clearly visible.
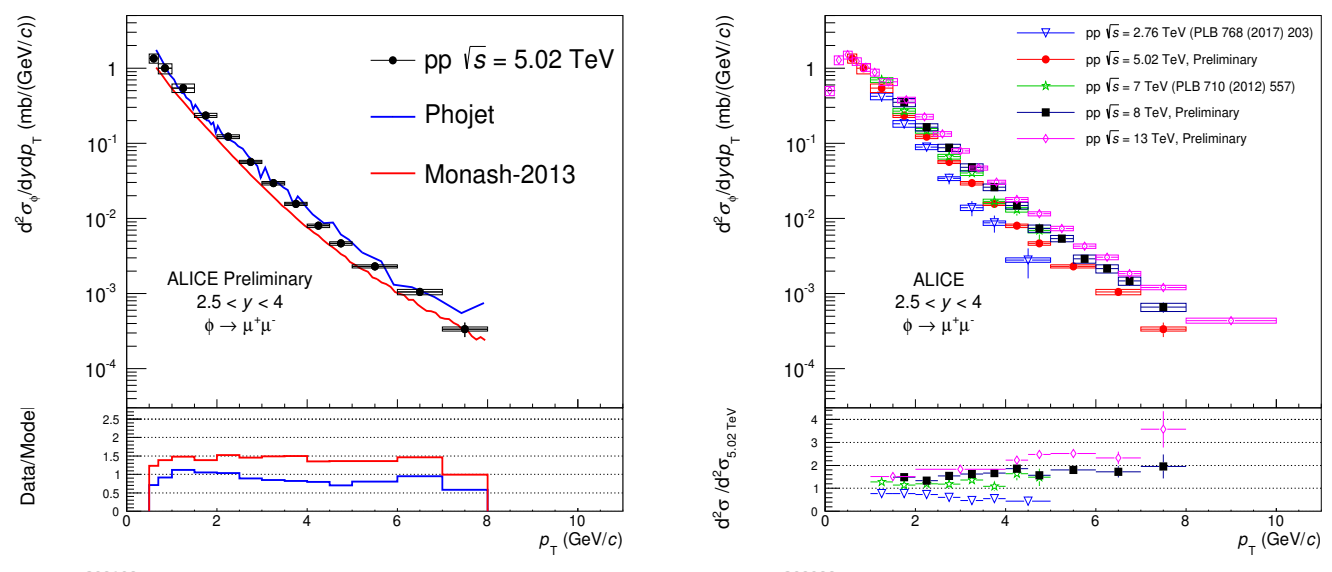

Figure 3: (colour online). Left: Production cross section of the $\phi$ meson as a function of $p_{\mathrm{T}}$ measured in pp collisions at $\sqrt{s}=5.02 \mathrm{TeV}$. Right: Energy dependence of the $\phi$-meson production in pp collisions.

\section{Summary}

The dielectron invariant-mass spectrum measured in $\mathrm{Pb}-\mathrm{Pb}$ collisions at $\sqrt{s_{\mathrm{NN}}}=2.76 \mathrm{TeV}$ is described by the contributions of known hadronic sources and thermal dielectrons with in-medium modified $\rho^{0}$ mesons. The sensitivity of this measurement is limited and prevents the analysis of 
a possible excess spectrum in the low-mass region and the study of charm and bottom modifications due to shadowing and energy loss effects. A suppression of dielectrons from heavy-flavor hadron decays is observed in the intermediate-mass range of the dielectron spectrum measured in $\mathrm{Pb}-\mathrm{Pb}$ collisions at $\sqrt{s_{\mathrm{NN}}}=5.02 \mathrm{TeV}$. The data are consistent with the cocktail that includes only shadowing effects for dielectrons from charm decays, indicating the limited sensitivity of this measurement to hot nuclear matter effects. A higher statistical precision is reached in the measurement of the $\phi$-meson production cross section in pp collisions at $\sqrt{s}=5.02 \mathrm{TeV}$ by including the data set from 2017. The systematic uncertainties are also reduced thanks to significant improvements in the signal extraction techniques.

\section{References}

[1] R. Rapp, Phys. Rev. C63 (2001) 054907.

[2] R. Rapp, Adv. High Energy Phys. 2013 (2013) 148253.

[3] S. Acharya et. al. (The ALICE Collaboration), JHEP 09 (2018) 64.

[4] S. Acharya et. al. (The ALICE Collaboration), Phys. Lett. B788 (2019) 505.

[5] K. Aamodt et. al. (The ALICE Collaboration), JINST 3 (2008) S08002.

[6] K. Aamodt et. al. (The ALICE Collaboration), Int. J. Mod. Phys. A29 (2014) 1430044.

[7] E. Abbas et. al. (The ALICE Collaboration), JINST 8 (2013) P10016.

[8] J. Alme et. al. (The ALICE Collaboration), Nucl. Instrum. Meth. A622 (2010) 316.

[9] K Aamodt et. al. (The ALICE Collaboration), JINST 5 (2010) P03003.

[10] A. Akindinov et. al. (The ALICE Collaboration), EPJ Plus 128 (2013) 4.

[11] S. Acharya et. al. (The ALICE Collaboration), arXiv:1807.00923 [nucl-ex].

[12] T. Song, W. Cassing, P. Moreau, E. Bratkovskaya, arXiv:1803.02698 [nucl-th].

[13] R. Arnaldi et. al. (The NA60 Collaboration), Phys. Rev. Lett. 96 (2006) 162302.

[14] S. Damjanovic et. al. (The NA60 Collaboration), Nucl. Phys. A783 (2007) 327.

[15] D. Adamova et. al. (The CERES Collaboration), Phys. Lett. B666 (2008) 425.

[16] A. Adare et. al. (The PHENIX Collaboration), Phys. Rev. C81 (2010) 034911.

[17] L. Adamczyk et. al. (The STAR Collaboration), Phys. Rev. Lett. 113 (2014) 049903.

[18] A. Adare et. al. (The PHENIX Collaboration), Phys.Rev. C93 (2016) 014904.

[19] R. Rapp et. al., Nucl. Phys. A979 (2018) 21.

[20] P. Skands, S. Carrazza, J. Rojo, arXiv:1404.5630 [hep-ph]

[21] R. Engel et. al., Phys. Rev. D52 (1995) 1459. 